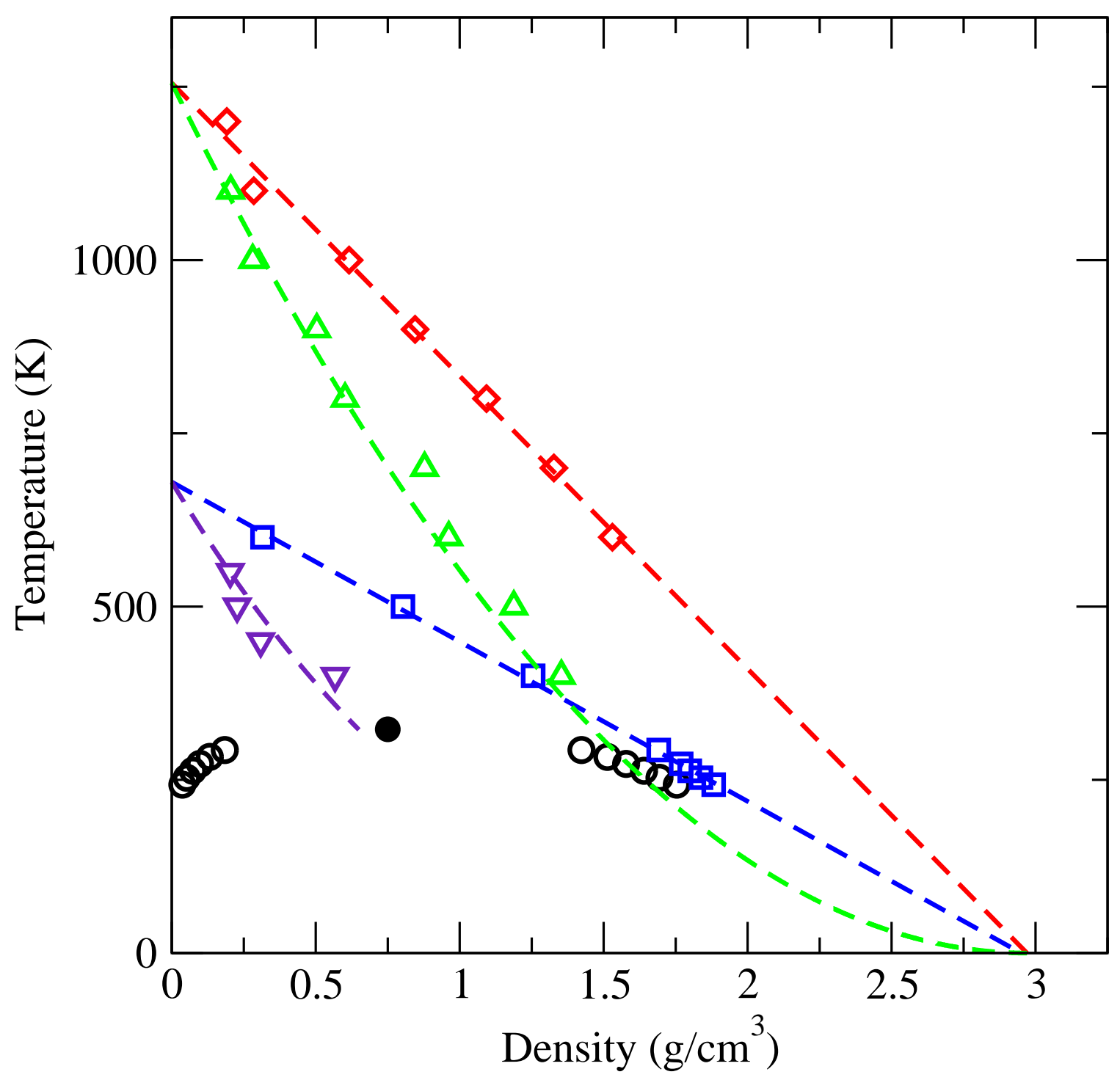




\title{
Ideality contours and thermodynamic regularities in supercritical molecular fluids.
}

\author{
Caroline Desgranges ${ }^{1}$, Abigail Margo ${ }^{1}$ and Jerome Delhommelle ${ }^{1 *}$ \\ ${ }^{1}$ Department of Chemistry, University of North Dakota, Grand Forks ND 58202
}

\begin{abstract}
Using Expanded Wang-Landau simulations, we calculate the ideality contours for 3 molecular fluids $\left(\mathrm{SF}_{6}, \mathrm{CO}_{2}\right.$ and $\left.\mathrm{H}_{2} \mathrm{O}\right)$. We analyze how the increase in polarity, and thus, in the strength of the intermolecular interactions, impacts the contours and thermodynamic regularities. This effect results the increase in the Boyle and $H$ parameters, that underlie the Zeno line and the curve of ideal enthalpy. Furthermore, a detailed analysis reveals that dipole-dipole interactions lead to much larger enthalpic contributions to the Gibbs free energy. This accounts for the much higher temperatures and pressures that are necessary for supercritical $\mathrm{H}_{2} \mathrm{O}$ to achieve ideal-like thermodynamic properties.
\end{abstract}

Keywords: Sulfur hexafluoride, Carbon dioxide, Water, Critical properties, Wang-Landau

\section{Introduction}

Supercritical fluids [1] have become increasingly important in environmentally benign separations and reactions $[2,3]$, and for new ways of processing materials [4]. This is because of their unusual physical properties including their high compressibility, their transport properties intermediated between gas and liquid and their high dissolving power [5]. Among the supercritical fluids used in these applications, carbon dioxide stands out as one of the most versatile [6]. Recent work has also highlighted the role played by supercritical water in nature e.g. in the Earth crust and mantle [7], and as a green

\footnotetext{
*Corresponding author. Email: jerome.delhommelle@und.edu
}

Preprint submitted to Chemical Physics Letters

June 6, 2016

(C) 2016. This manuscript version is made available under the Elsevier user license http://www.elsevier.com/open-access/userlicense/1.0/ 
solvent for the catalytic conversion of biomass into fuel [8]. Processes aiming to capture and store $S F_{6}$, a potent greenhouse gas that is supercritical under ambient conditions, are also currently being developed [9]. However, there are still significant gaps in our understanding of the thermophysical properties of supercritical fluids $[10,11]$.

Recent work has shown the emergence of several remarkable thermodynamic contours $[12,13]$ that could help bridge this gap in knowledge. The existence and similarity of such contours, which span the supercritical domain, would allow to build a correspondence between the supercritical fluids of different compounds, in the spirit of the law of corresponding states that connects the binodal and critical point for a wide range of substances. The aim of this work is to study supercritical fluids that are found in a number of technological and environmental applications and to provide a full picture of their thermodynamics in the supercritical domain of their phase diagram. We focus specifically on three molecular fluids: $S F_{6}$, for which we parametrize a new force field, carbon dioxide modeled with the TraPPE force field [14] and water modeled with the SPC/E potential [15]. We study the behavior of these molecular fluids along a number of remarkable contours, known as the Zeno line (where the compressibility factor is equal to 1 ), the $H$ line (where the enthalpy is the same of that of an ideal gas), the $S_{0}$ line (where the isothermal compressibility, or, equivalently, the structure factor at zero wave vector $S_{0}$ is equal to zero) and the $H_{\min }$ line (the line of minima for the enthalpy isotherms). Recent work has shown the peculiar behavior of supercritical fluids along these lines (most notably the straightness of the Zeno line and of the $H$ line for the Lennard-Jones (LJ) fluid [13]). However, the behavior of molecular fluids along these contours is still largely unknown. To elucidate this point, we determine the thermodynamics properties of supercritical $\mathrm{SF}_{6}, \mathrm{CO}_{2}$ and $\mathrm{H}_{2} \mathrm{O}$, analyze the impact of the type of intermolecular interactions and of their strength on the thermodynamic contours and regularities, and interpret the results in terms of their enthalpic and entropic contributions to the Gibbs free energy.

The paper is organized as follows. In the next section, we briefly define the thermodynamic contours and regularities studied in this work. Then, in the next section, we present the simulation method, molecular models and technical details. In particular, we discuss how the recently developed Expanded Wang-Landau simulations $[16,17,18,19]$ are applied to determine the loci for the thermodynamic contours in the supercritical region of the phase diagram. The advantage of using EWL simulations is twofold: (i) the 
same method is consistently used to generate the data for all systems and contours, (ii) the conditions needed to achieve ideal-like behavior often involve very high temperatures/pressures, with few, if any, experimental data or equations of state covering that region of the phase diagram. We determine the properties of supercritical fluids and compare the behavior of the different fluids to characterize the effect of the intermolecular interactions on the thermodynamic contours before drawing the main conclusion of this work in the last section.

\section{Contours and regularities}

Recent work has shown that several thermodynamic regularities established on the van der Waals (vdW) equation can be extended to real substances [13]. First, the contour $Z=P / \rho T=1$ (where $Z$ is the compressibility factor, $P$ the pressure, $\rho$ the number density and $T$ the temperature) follows a straight line in the density-temperature plane for the vdW equation, for model systems [20], water [21] and several other compounds [22, 23, 24]. Second, the $H$ contour (curve of ideal enthalpy) is a straight line for the vdW equation [12] and for the LJ fluid [13]. However, the shape of the $H$ line has yet to be established for molecular systems. We also consider two other contours, the $S_{0}$ and $H_{\text {min }}$ lines. The $S_{0}$ line is the curve of maxima for the isothermal compressibility where $\left(\partial^{2} P / \partial \rho^{2}\right)_{T}=0$, and extends from the critical point into the supercritical region of the phase diagram. It corresponds to the line of density fluctuation maxima, that delimits the ridges in the supercritical region [11, 25, 26], and, as such, is connected with the possible separation of the supercritical region into gas-like and liquid-like phases [26]. The $H_{\text {min }}$ line is defined as the line of minima for the enthalpy isotherms. As discussed in [13], the $H_{m i n}$ line underlies the line of ideal enthalpy, as its limiting values, both at zero density and at zero temperature, coincide with that of the $H$ line. The impact of the intermolecular interactions on the shape of these contours is unknown. Elucidating the thermodynamics of molecular fluids along these 4 contours will be a first step towards establishing a correspondence between supercritical fluids, as these contours span the supercritical region of the phase diagram. 


\section{Simulation Methods}

\subsection{Expanded Wang-Landau Simulations}

We use the Expanded Wang-Landau (EWL) simulation method [16, 17, $18,19]$. This approach takes advantage of a flat histogram sampling technique, the Wang-Landau scheme [27, 28, 29, 30, 31, 32], combined with an expanded ensemble approach [33, 34, 35, 36, 37, 38, 39, 40, 41, 42, 43], in which the difficult insertion/deletion of entire molecules is achieved by varying the size of a fractional molecule (more details are given in $[16,17,18,19]$ ). The advantage of the EWL method is that it only requires the temperature and volume as input parameters, and that a single EWL run is required, for a given temperature, to obtain the properties for all densities. The simulation consists in evaluating the partition function $\Theta(\mu, V, T)$ in the grand-canonical ensemble:

$$
\Theta(\mu, V, T)=\sum_{N=0}^{\infty} Q(N, V, T) \exp (\beta \mu N)
$$

with

$$
Q(N, V, T)=\frac{V^{N}}{N ! \Lambda^{3 N}} \int \exp (-\beta U(\Gamma)) d \Gamma
$$

Having an accurate estimate for the grand-canonical partition function allows, in turn, for the calculation of all thermodynamic properties of the system, particularly those difficult to compute such as e.g. the Gibbs free energy and the entropy.

\subsection{Molecular Models}

$S F_{6}$ is modeled with a set of six LJ sites, each site standing for a $F$ atom. Interactions between two LJ sites $(i, j)$ are given by

$$
\phi\left(r_{i j}\right)=4 \epsilon\left[\left(\frac{\sigma}{r_{i j}}\right)^{12}-\left(\frac{\sigma}{r_{i j}}\right)^{6}\right]
$$

where $r_{i j}$ is the distance between the two atoms, and $\epsilon$ and $\sigma$ are the energy and size parameters for the potential. We use a distance between the center of the molecule ( $S$ atom) and each of the $F$ atom of $1.62 \AA$. Optimizing the values for the LJ parameters to best reproduce the vapor-liquid densities at coexistence leads to the following set of parameters: $\epsilon / k_{B}=78$ and $\sigma=$ 

and of a full (regular) molecule is obtained by scaling the energy and size parameters by $(l / M)^{1 / 3}$ and $(l / M)^{1 / 4}$, respectively, where $M$ is the number of stages in which the insertion/deltion steps are divided and $l$ is the current value for the stage number of the fractional molecule [16]. In addition, the size of the fractional molecule (i.e. the bond length between $S$ and $F$ ) is scaled by $(l / M)^{1 / 4}$ to ensure that the fractional molecule is smaller than a full molecule.

$\mathrm{CO}_{2}$ is modeled with the TraPPE potential [14]. Each molecule is described as a distribution of three LJ sites and three point charges (one on each atom) with the interaction between atoms $(i, j)$ as

$$
\phi\left(r_{i j}\right)=4 \epsilon_{i j}\left[\left(\frac{\sigma_{i j}}{r_{i j}}\right)^{12}-\left(\frac{\sigma_{i j}}{r_{i j}}\right)^{6}\right]+\frac{q_{i} q_{j}}{4 \pi \epsilon_{0} r_{i j}}
$$

To model the interaction between an atom of the fractional molecule with an atom of a full molecule we scale the interaction parameters $\epsilon_{i j}, \sigma_{i j}$ and the product $q_{i} q_{j}$ by $(l / M)^{1 / 3},(l / M)^{1 / 4}$ and $(l / M)^{1 / 3}$, respectively. We scale the size of the fractional molecule (i.e. the bond length between $C$ and $O$ ) as above, in the case of $S F_{6}$.

Finally, we use the SPC/E force field [15] to model $\mathrm{H}_{2} \mathrm{O}$. It relies on the same functional form for the interaction potential as Eq. 4. We add that we also use the same scaling both for the interaction between the fractional molecule and a full molecule and for the size of the fractional molecule as for $\mathrm{SF}_{6}$ and $\mathrm{CO}_{2}$.

\subsection{Technical Details}

EWL simulations consist of the following Monte Carlo (MC) steps for all molecules: $37.5 \%$ of the attempted MC moves are translations of a single molecule (full or fractional), 37.5\% are rotations of a single molecule (full or fractional) and the $25 \%$ remaining moves are changes in $(N, l)$ values. The number of stages $M$ is set to 100 , the starting value for the convergence factor $f$ in the iterative Wang-Landau scheme to $e$, its final value to $10^{-8}$, with each $(N, l)$ value being visited at least 1000 times for a given value of $f$. Simulations are carried out on cubic cells for all systems, with periodic boundary conditions, and a box length of $30 \AA$. . LJ and quadrupolar interactions are calculated using a spherical cutoff set to half the box length. The usual tail corrections are applied beyond that cutoff distance for the LJ part 
of the potential. In the case of $\mathrm{H}_{2} \mathrm{O}$, Ewald sums are used to calculate the electrostatic part of the potential energy (with the same parameters as in ref. [18]).

\section{Results and Discussion}

\section{1. $S F_{6}$}

We start by discussing the results obtained for $S F_{6}$. The left panel of Fig. 1 shows the grand-canonical partition function $\Theta(\mu, V, T)$ (top left of Fig. 1) as well as the associated $Q(N, V, T)$ functions (bottom left). The plot of $\log \Theta(\mu, V, T)$ against the chemical potential $\mu$ provides a direct insight into the nature of the fluid (subcritical or supercritical) as a function of the thermodynamic conditions. At low temperature (below $293 \mathrm{~K}$ ), the plots for $\log \Theta$ exhibit a sharp change beyond a threshold value for $\mu$, corresponding to the vapor $\rightarrow$ liquid transition. As temperature increases, $\log \Theta$ displays a smoother increase with $\mu$, showing that the fluid is now supercritical. As shown in Eq. 1, the grand-canonical partition function is a sum of the $Q(N, V, T)$ functions (weighted by the $\exp (\mu N)$. The differences observed for $\Theta$ as a function of $T$ are therefore directly connected to those seen, for different $T$, on $Q(N, V, T)$ as a function of $N$, since the slope of the $\log Q$ plot against $N$ is related to $\mu$. This means that, at low temperature, when the slope for $\log Q$ is very large, the transition towards the high density phase (or equivalently the sharp increase in $\log \Theta$ ) will occur for larger values of $\mu$. Moreover, for $(T>400 \mathrm{~K}$, the curves for $\log Q$ are flatter, confirming that the fluid is supercritical.

Knowing $\Theta(\mu, V, T)$ and $Q(N, V, T)$ allows us to determine the loci for the thermodynamic contours. We define the number distribution $p(N)$ as

$$
p(N)=\frac{Q(N, V, T) \exp (\beta \mu N)}{\Theta(\mu, V, T)}
$$

This yields the conditions of coexistence, obtained by numerically solving the following equation:

$$
\sum_{N=0}^{N_{b}} p(N)=\sum_{N_{b}}^{\infty} p(N)
$$

where $N_{b}$ is the point at which the function $p(N)$ reaches its minimum, and the left hand side and the right hand side of the equation correspond to the 
Figure 1: EWL results for $S F_{6}$. (Top left) $\log \Theta(\mu, V, T)$ against $\mu$ for $243 \mathrm{~K}$ (black), $243 \mathrm{~K}$ (black), $263 \mathrm{~K}$ (red), $293 \mathrm{~K}$ (green), $400 \mathrm{~K}$ (blue), $600 \mathrm{~K}$ (indigo), $800 \mathrm{~K}$ (brown), $1000 \mathrm{~K}$ (magenta) and $1200 \mathrm{~K}$ (cyan). (Bottom left) $\log Q(N, V, T)$ against $N$ (same legend as for $\log \Theta)$. (Top right) $p(N)$ at coexistence for $293 \mathrm{~K}$. (Bottom right) $p(N)$ along the $S_{0}$ line (indigo), $Z$ line (blue), $H_{\min }$ line (green) and $H$ line (red) at $600 \mathrm{~K}$.

probability of the vapor and of the liquid phase, respectively. We provide in the top right corner of Fig. 1 the curve for $p(N)$ obtained at coexistence for $T=293 K$. From there, the densities at coexistence can be readily calculated to obtain the binodal curve. $p(N)$ is also used to locate the Zeno line by solving the $Z=1$ equation

$$
P \bar{V} / R T=\log \Theta(\mu, V, T) / \bar{N}=1
$$

where $\bar{V}$ is the reciprocal density and $\bar{N}=\sum N p(N)$ is the average number of particles in the system. The corresponding point of the Zeno line at $T=600 \mathrm{~K}$ is shown in bottom right corner of Fig. 1. For the $H$ line and $H_{\text {min }}$ contour, we evaluate the enthalpy from $p(N)$ as

$$
H=U+P V=\frac{\sum\left(E_{\text {pot }}(N)+\frac{3}{2} k_{B} T\right) p(N)}{\sum p(N)}+k_{B} T \log \Theta(\mu, V, T)
$$

where $E_{p o t}(N)$ is the potential energy per molecule for a system of $N$ molecules. The $H$ line is then obtained by solving $\bar{H}=4 R T$ and the $H_{\min }$ contour by minimizing $H$ reaches at fixed $T$. Examples of $p(N)$ for the $H$ and $H_{\text {min }}$ 

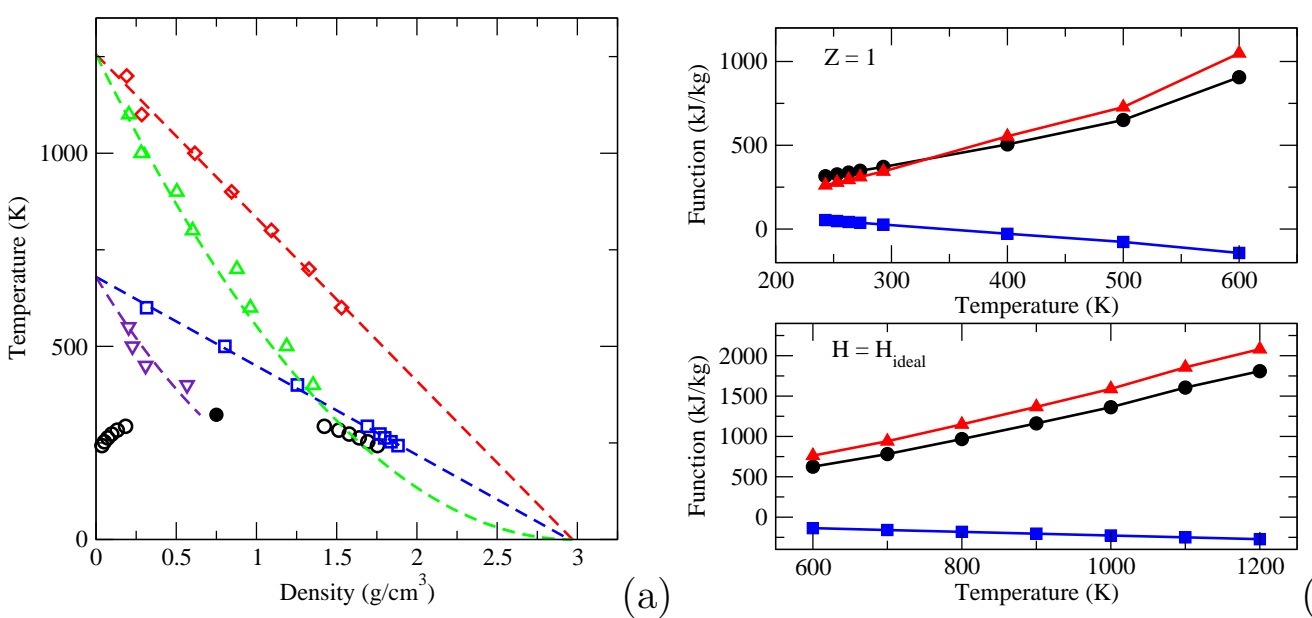

(b)

Figure 2: (a) EWL results for the coexistence curve of $S F_{6}$ (open circles), critical point (filled circle), $S_{0}$ line (indigo), $Z$ line (blue), $H_{\min }$ line (green) and $H$ line (red). (b) Thermodynamic functions along the $Z$ line (top) and the $H$ line (bottom), with $-G$ (circles), $-H$ (squares) and $T S$ (triangles).

points at $T=600 \mathrm{~K}$ are also provided in Fig. 1 . The $S_{0}$ contour is obtained by differentiating numerically $P$ twice with respect to the number density and finding $\mu$ such that $\left(\partial^{2} P / \partial \rho^{2}\right)_{T}=0$, with the corresponding $p(N)$ at $T=600 K$ shown in Fig. 1.

We show in Fig. 2(a) the binodal as well as the critical point obtained from the EWL results for the newly parameterized force field. We use a scaling law, with a critical exponent of 0.326 , to determine the critical temperature and the law of rectilinear diameter for the critical density (see Table 1). The results are in good agreement with the experimental results [44] $\left(T_{c}=\right.$ $318.7 \mathrm{~K}$ and $\left.\rho_{c}=0.737 \mathrm{~g} / \mathrm{cm}^{3}\right)$. Also shown in Fig. 2(a) are the EWL contours for $S F_{6}$. As for the LJ fluid [13], the straightness of the $Z$ line and of the $H$ line is conserved throughout the phase diagram, both in the subcritical and in the supercritical regimes. Specifically, linear fits to the EWL results lead to an Average Absolute Relative Deviation $(A A R D)$ error $\left(A A R D(\%)=\frac{1}{N} \sum_{i}\left|\frac{T_{F i t}-T_{\text {sim }}}{T_{\text {sim }}}\right|\right)$ of $1.0 \%$ for the $Z$ line and to an error of $1.1 \%$ for the $H$ line. The fits yield the Boyle parameters, i.e. the Bole temperature $T_{B}$ and Boyle density $\rho_{B}$ (intercepts for the $Z$ line), as well as the $H$ parameters, $T_{H}$ and $\rho_{H}$ (intercepts for the $H$ line), given in Table 1. The $T_{B}: T_{H}$ ratio for $S F_{6}(0.54)$ is in good agreement with that obtained 
for the LJ fluid (0.53). Similarly, the $\rho_{B}: \rho_{H}$ ratio for $S F_{6}(0.99)$ is close to that of LJ (0.92) and the shapes for the $H_{\min }$ and $S_{0}$ contours are similar to the LJ contours. We then model the $H_{\min }$ line with a quadratic law, $T_{H_{\min }(\rho)}=T_{H}\left(1-\rho / \rho_{H}\right)^{2}$, which is exact for $\mathrm{vdW}$ [13]. The simulation results are accurately modeled by this law (see Fig. 2(a)), with an error of $6.8 \%$. Similarly, we model the results for the $S_{0}$ line with a cubic law $T_{S_{0}}(\rho)=T_{B}\left(1-\rho / \rho_{B}\right)^{3}$ and obtain an overall fair agreement, with an AARD of $21 \%$, which is reasonable given the numerical uncertainties arising from the successive differentiations involved to obtain the $S_{0}$ line and the fact that this law does not allow for any adjustable parameter. The similarity between $S F_{6}$ and the LJ/vdW systems can be attributed to the type of intermolecular interaction that exists in $S F_{6}$, specifically, to the nonpolar nature of $S F_{6}$ and the absence of long-range interactions.

Table 1: Boyle, $\mathrm{H}$ and critical parameters for $\mathrm{SF}_{6}, \mathrm{CO}_{2}$ and $\mathrm{H}_{2} \mathrm{O}$ ( $\mathrm{T}$ are given in $\mathrm{K}$, and $\rho$ in $\left.\mathrm{g} / \mathrm{cm}^{3}\right)$.

\begin{tabular}{|c|c|c|c|c|c|c|}
\hline \hline & $T_{B}$ & $\rho_{B}$ & $T_{H}$ & $\rho_{H}$ & $T_{c}$ & $\rho_{c}$ \\
\hline \hline $\mathrm{SF}_{6}$ & $680 \pm 6$ & $2.95 \pm 0.07$ & $1255 \pm 15$ & $2.97 \pm 0.15$ & $323 \pm 3$ & $0.75 \pm 0.01$ \\
$\mathrm{CO}_{2}$ & $683 \pm 3$ & $1.84 \pm 0.03$ & $1255 \pm 7$ & $1.89 \pm 0.04$ & $306 \pm 3$ & $0.47 \pm 0.01$ \\
$\mathrm{H}_{2} \mathrm{O}$ & $1599 \pm 19$ & $1.34 \pm 0.05$ & $2722 \pm 17$ & $1.84 \pm 0.08$ & $641 \pm 5$ & $0.31 \pm 0.01$ \\
\hline \hline
\end{tabular}

We now turn to the variations of the properties along the $Z$ line and the $H$ line. Let us consider first the case when $S F_{6}$ behaves as an ideal gas from a Zeno standpoint. The top of Fig. 2(b) shows that the entropic contribution to the $G$ is predominant throughout the temperature range. However, we observe a crossover between the $-G$ curve and the $T S$ curve as the fluid goes from the subcritical to the supercritical regime. This crossover occurs with a changing sign of $H$ when going from subcritical temperatures (where $H$ is negative as a result of the strong attractive intermolecular interactions) to supercritical temperatures (where, as a result of the decrease in density, the contribution from the attractive intermolecular interactions becomes negligible). Considering now the case where $S F_{6}$ exhibits the same enthalpy as an ideal gas, we do not observe any crossover between $T S$ and $G$ since $H$ always remains positive.

\section{2. $\mathrm{CO}_{2}$}

The results for the phase diagram and contours for $\mathrm{CO}_{2}$ are shown in Fig. 3(a). The results are in good agreement with the experimental data [44] 

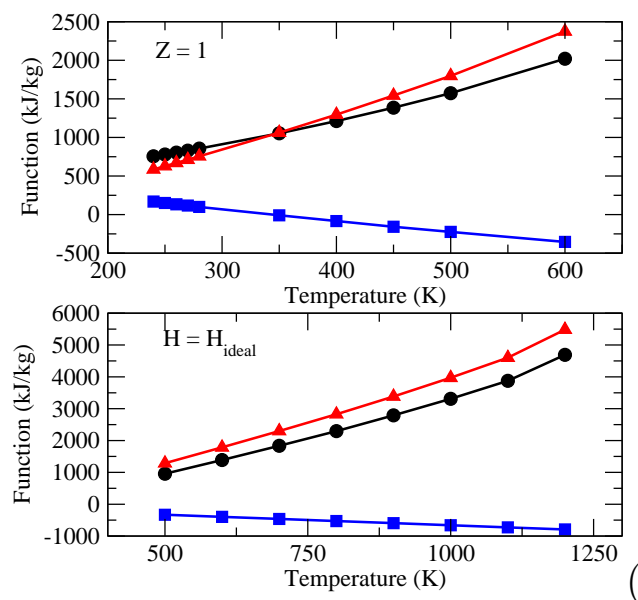

Figure 3: (a) EWL results for the coexistence curve of $\mathrm{CO}_{2}$ (open circles), critical point (filled circle), $S_{0}$ line (indigo), $Z$ line (blue), $H_{\min }$ line (green) and $H$ line (red). (b) Thermodynamic functions along the $Z$ line (top) and the $H$ line (bottom), with $-G$ (circles), $-H$ (squares) and $T S$ (triangles).

at coexistence and for the critical properties $\left(T_{c}=304.2 \mathrm{~K}\right.$ and $\rho_{c}=$ $0.47 \mathrm{~g} / \mathrm{cm}^{3}$ ). We fit the $Z$ and $\mathrm{H}$ lines for $\mathrm{CO}_{2}$ to a straight line. This shows that the quadrupolar interactions do not impact the features of the contours. The straightness of the lines, in particular of the $Z$ line holds well with an $A A R D$ of $0.5 \%$. The EWL results for $C_{2}$ are in very good agreement with those extrapolated from the experimental data on low temperature liquids [24]. The EWL $T_{c}: T_{B}$ ratio $(0.44)$ and $\rho_{c}: \rho_{B}$ ratio $(0.26)$ are close to those (0.42 and 0.26$)$ from [24]. We also observe that the straightness of the $H$ line also holds, with a very low $A A R D$ for the $H$ line of $0.8 \%$. The $T_{B}: T_{H}$ ratio $(0.54)$ is similar to $S F_{6}(0.54)$ and the $\rho_{B}: \rho_{H}$ ratio $(0.97)$ is close close to the value found for $S F_{6}$ (0.99). This shows the limited impact of the quadrupolar interactions on the straightness of the ideality contours. We then use the $H$ and Boyle parameters to model the EWL results for the $H_{\text {min }}$ line and for the $S_{0}$ line. We find that both the two quadratic (for $H_{\text {min }}$ ) and cubic (for $S_{0}$ ) laws adequately account for the behavior of $\mathrm{CO}_{2}$, as shown in Fig. 3(a). We find an AARD of $3.6 \%$ for the $H_{\text {min }}$ line and of $24 \%$ for the $S_{0}$ line with the two laws proposed on the basis of the vdW equation and with no adjustable parameter. This further establishes that $\mathrm{CO}_{2}$, like $S F_{6}$, has a behavior close to a vdW/LJ system in the supercritical region. 
We finally calculate the ratios $\rho_{c}\left(C O_{2}\right): \rho_{c}\left(S F_{6}\right), \rho_{B}\left(C O_{2}\right): \rho_{B}\left(S F_{6}\right)$ and $\rho_{H}\left(C_{2}\right): \rho_{H}\left(S F_{6}\right)$ and find that these are all roughly of 0.62 . Similarly, the ratios for the critical, Boyle and $H$ temperatures for the two compounds are all close to 1 . These results show that a correspondence between the supercritical properties of nonpolar molecular fluids can be established.

Focusing now on the properties along the $Z$ line, we find a similar behavior for $\mathrm{CO}_{2}$ similar to $S F_{6}$. Over the same temperature interval (240 $\mathrm{K}$ $600 \mathrm{~K}), G$ undergoes an almost three-fold decrease for both fluids (2.7 for $\mathrm{CO}_{2}$, compared to 2.9 for $S F_{6}$ ), resulting from a combined decrease in the enthalpic contribution, with a change in sign in the top of the temperature range, $\left(\times 2.1\right.$ for $\mathrm{CO}_{2}$ and $\times 2.6$ for $\left.S F_{6}\right)$ and an increase in the TS $(\times 4.1$ for $\mathrm{CO}_{2}$ and $\times 4.0$ for $S F_{6}$ ). The results for the $H$ line show similar trends for both compounds. Considering the same temperature interval for the two fluids $(600 \mathrm{~K}-1200 \mathrm{~K})$, we find a decrease in the $G$ by a factor of 3.4 for $\mathrm{CO}_{2}$ (as compared to 2.9 for $S F_{6}$ ), resulting from a decrease in enthalpy by a factor of 2 for $\mathrm{CO}_{2}$ (same as for $S F_{6}$ ) and an increase in the entropic term by a factor of 3.1 for $\mathrm{CO}_{2}$ (compared to 2.7 for $S F_{6}$ ). These similarities further show the quadrupolar interactions in $\mathrm{CO}_{2}$ have a moderate effect on the thermodynamics of the supercritical fluid.

\section{3. $\mathrm{H}_{2} \mathrm{O}$}

We show in Fig. 4(a) the EWL results for the binodal and contours for $\mathrm{H}_{2} \mathrm{O}$, with a critical temperature $T_{c}=641 \mathrm{~K}$ in good agreement with the experimental value of $647 K$ [44] and previous work [45], and a critical density $\rho_{c}=0.31 \mathrm{~g} / \mathrm{cm}^{3}$ close to the experiment [44] $\left(0.322 \mathrm{~g} / \mathrm{cm}^{3}\right)$. The EWL results reveal that the polar nature of $\mathrm{H}_{2} \mathrm{O}$ impacts significantly the fluid properties along the $Z$ line. Specifically, we find an $A A R D$ error of $3.7 \%$, greater than for the nonpolar fluid $S F_{6}$ and for the quadrupolar fluid $\mathrm{CO}_{2}$. However, the linear fit still describes well the $Z$ line and allows us to determine the Boyle parameters for $\mathrm{H}_{2} \mathrm{O}$ (see Table 1). We then fit the $H$ line. Given that low temperature results for the $H$ line are not accessible (since they lie within the domain of stability of the solid), the linear fit relies on points obtained at high temperatures and yields the $H$ parameters shown in Table 1, with an $A A R D$ of $0.3 \%$. While the $T_{B}: T_{H}$ ratio for $\mathrm{H}_{2} \mathrm{O}$ is reasonably close to that obtained for other fluids $\left(0.59\right.$ for $\mathrm{H}_{2} \mathrm{O}$ compared to 0.54 for the $S F_{6}$ ), the Boyle and $H$ densities are markedly different, with a decrease in

$\rho_{B}: \rho_{H}$ ratio which decreases down to 0.73 for $\mathrm{H}_{2} \mathrm{O}$ from $0.97\left(\mathrm{CO}_{2}\right)$ and 0.99 $\left(S F_{6}\right)$. This suggests that the dipolar interactions become predominant at 

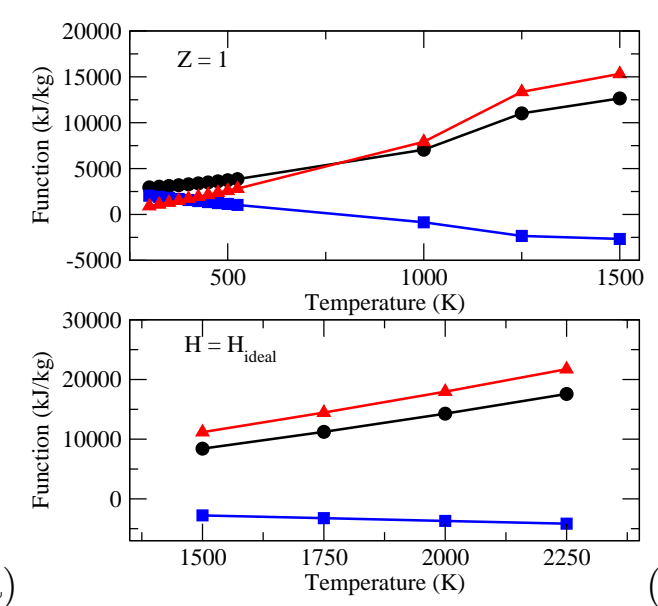

(b)

Figure 4: (a) EWL results for the coexistence curve of $\mathrm{H}_{2} \mathrm{O}$ (open circles), critical point (filled circle), $S_{0}$ line (indigo), $Z$ line (blue), $H_{\min }$ line (green) and $H$ line (red). (b) Thermodynamic functions along the $Z$ line (top) and the $H$ line (bottom), with $-G$ (circles), $-H$ (squares) and $T S$ (triangles).

low temperatures and that very large pressures/densities are required for the enthalpy of $\mathrm{H}_{2} \mathrm{O}$ to become equivalent to that of an ideal gas. The behaviors of the $\mathrm{H}_{\min }$ and $\mathrm{S}_{0}$ lines strongly depart from that found for $\mathrm{SF}_{6}$ and $\mathrm{CO}_{2}$, with large deviations from the quadratic law for $H_{\min }$ (AARD of 21\%) and from the cubic law for $S_{0}$ (AARD of $35 \%$ ). The two laws completely miss the qualitative features of these two lines in the low temperatures/large densities regime. For the $H_{\text {min }}$ line, the quadratic law only models well the behavior of the $S P C E / E$ model for temperatures above $1500 \mathrm{~K}$, while the cubic law never really captures the $S_{0}$ line and the critical point. Furthermore, the correspondence seen in the case of $\mathrm{SF}_{6}$ and $\mathrm{CO}_{2}$ does not apply to $\mathrm{H}_{2} \mathrm{O}$, as e.g. the ratio of the $\mathrm{H}$ densities of $\mathrm{H}_{2} \mathrm{O}$ and $\mathrm{CO}_{2}$ is 0.97 , while the ratio of the Boyle densities for the two compounds is 0.73 .

Fig. 4(b) shows the variations of the properties along the $Z$ line. A notable difference with $\mathrm{SF}_{6}$ and $\mathrm{CO}_{2}$ is the relative importance of the two contributions (enthalpic and entropic) to $G$. The enthalpic contribution amounts to up to $70 \%$ of the $G$ for $\mathrm{H}_{2} \mathrm{O}$ at the beginning of the $Z$ line, while it is only of $17 \%$ in the case of $\mathrm{SF}_{6}$ and of $22 \%$ for $\mathrm{CO}_{2}$. The stronger intermolecular interactions in $\mathrm{H}_{2} \mathrm{O}$ imply that larger temperatures need to be applied to reach ideality as shown by the larger value for $T_{B}$ for $H_{2} O(1599.4 \mathrm{~K})$. Simi- 
lar conclusions hold for the $H$ line, with the dipolar interactions in resulting in a larger $T_{H}$.

\section{Conclusions}

In this work, we determine the ideality contours and the Boyle, critical and $\mathrm{H}$ parameters of $\mathrm{SF}_{6}, \mathrm{CO}_{2}$ and $\mathrm{H}_{2} \mathrm{O}$. For $\mathrm{SF}_{6}$, we parametrize a 6 -site force field that yelds critical properties in excellent agreement with the experiment. We find the straightness of the $Z$ and $H$ lines also holds for a nonpolar molecular fluid like $\mathrm{SF}_{6}$. Then, for $\mathrm{CO}_{2}$, we find that quadrupolar interactions have a moderate impact on the thermodynamic properties of the supercritical fluid, and that the straightness of the $Z$ and $H$ lines remains valid across the phase diagram. Similarly, the behavior of the $H_{\min }$ line and of the $S_{0}$ line is accurately modeled by the simple polynomial laws that hold for the vdW equation. The results also show that a correspondence between the supercritical properties of nonpolar molecular fluids can be established since two nonpolar compounds exhibit the same ratio for their respective Boyle and $\mathrm{H}$ properties. The case of $\mathrm{H}_{2} \mathrm{O}$ exhibit departures from this behavior, with large deviations in the $Z$ and $H$ lines from linear fits. The same conclusions apply for the $H_{\text {min }}$ and $S_{0}$ lines, which exhibit strong departures from a vdW-like behavior, and show that the correspondence found between the supercritical properties of nonpolar fluids does not apply to $\mathrm{H}_{2} \mathrm{O}$. This change in behavior can be attributed to the strong dipole-dipole interactions in $\mathrm{H}_{2} \mathrm{O}$, which lead to a larger contribution of the enthalpic term to the Gibbs free energy and to higher temperatures/densities to achieve ideality. Further work, using water models developed for very high temperature/pressure conditions [46], would help refine these findings.

Acknowledgements Partial funding for this research was provided by NSF through CAREER award DMR-1052808.

\section{References}

[1] E. Kiran, P. G. Debenedetti, C. J. Peters, Supercritical Fluids: Fundamentals and Applications, volume 366, Springer Science \& Business Media, 2012.

[2] P. G. Jessop, T. Ikariya, R. Noyori, Chem. Rev. 99 (1999) 475-494.

[3] A. Baiker, Chem. Rev. 99 (1999) 453-474. 
[4] C. A. Eckert, Nature 383 (1996) 313-318.

[5] J. W. Tom, P. G. Debenedetti, J. Aerosol Sci. 22 (1991) 555-584.

[6] X. Han, M. Poliakoff, Chem. Soc. Rev. 41 (2012) 1428-1436.

[7] R. Kessel, P. Ulmer, T. Pettke, M. Schmidt, A. Thompson, Earth Planet. Sci. Lett. 237 (2005) 873-892.

[8] A. A. Peterson, F. Vogel, R. P. Lachance, M. Fröling, M. J. Antal Jr, J. W. Tester, Energy Environ. Sci. 1 (2008) 32-65.

[9] I. Senkovska, E. Barea, J. A. R. Navarro, S. Kaskel, Micropor. Mesopor. Mat. 156 (2012) 115-120.

[10] V. Brazhkin, Y. D. Fomin, A. Lyapin, V. Ryzhov, E. Tsiok, K. Trachenko, Phys. Rev. Lett. 111 (2013) 145901.

[11] V. Brazhkin, V. Ryzhov, J. Chem. Phys. 135 (2011) 084503.

[12] V. Nedostup, High Temperature 51 (2013) 72-78.

[13] E. Apfelbaum, V. Vorob'ev, J. Phys. Chem. B 117 (2013) 7750-7755.

[14] J. J. Potoff, J. I. Siepmann, AIChE Journal 47 (2001) 1676-1682.

[15] H. Berendsen, J. Grigera, T. Straatsma, Journal of Physical Chemistry 91 (1987) 6269-6271.

[16] C. Desgranges, J. Delhommelle, J. Chem. Phys. 136 (2012) 184107.

[17] C. Desgranges, J. Delhommelle, J. Chem. Phys. 136 (2012) 184108.

[18] C. Desgranges, J. Delhommelle, J. Chem. Phys. 140 (2014) 104109.

[19] C. Desgranges, J. Delhommelle, J. Chem. Phys. 144 (2016) 124510.

[20] E. Apfelbaum, V. Vorob'ev, J. Chem. Phys. 130 (2009) 214111.

[21] M. C. Kutney, M. T. Reagan, K. A. Smith, J. W. Tester, D. R. Herschbach, J. Phys. Chem. B 104 (2000) 9513-9525.

[22] E. Apfelbaum, V. Vorob'ev, J. Phys. Chem. B 112 (2008) 13064-13069. 
[23] E. Apfelbaum, V. Vorob'ev, J. Phys. Chem. B 113 (2009) 3521-3526.

[24] E. Apfelbaum, V. Vorob'ev, J. Phys. Chem. B 119 (2015) 8419-8424.

[25] K. Nishikawa, K. Kusano, A. A. Arai, T. Morita, The Journal of chemical physics 118 (2003) 1341-1346.

[26] V. V. Brazhkin, A. G. Lyapin, V. N. Ryzhov, K. Trachenko, Y. D. Fomin, E. N. Tsiok, Physics-Uspekhi 55 (2012) 1061.

[27] F. Wang, D. Landau, Phys. Rev. Lett. 86 (2001) 2050-2053.

[28] M. S. Shell, P. G. Debenedetti, A. Z. Panagiotopoulos, Phys. Rev. E 66 (2002) 056703.

[29] Q. Yan, R. Faller, J. J. de Pablo, J. Chem. Phys. 116 (2002) 8745-8750.

[30] G. Gazenmüller, P. J. Camp, J. Chem. Phys. 127 (2007) 154504.

[31] C. Desgranges, J. Delhommelle, J. Chem. Phys. 130 (2009) 244109.

[32] T. Aleksandrov, C. Desgranges, J. Delhommelle, Fluid Phase Equil. 287 (2010) 79-83.

[33] F. Escobedo, J. J. de Pablo, J. Chem. Phys. 105 (1996) 4391.

[34] A. P. Lyubartsev, A. A. Martsinovski, S. V. Shevkunov, P. N. VorontsovVelyaminov, J. Chem. Phys. 96 (1992) 1776-1783.

[35] M. Muller, W. Paul, J. Chem. Phys. 100 (1994) 719-724.

[36] W. Shi, E. J. Maginn, J. Chem. Theory Comp. 3 (2007) 1451-1463.

[37] J. K. Singh, J. R. Errington, J. Phys. Chem. B 110 (2006) 1369-1376.

[38] F. A. Escobedo, F. J. Martinez-Veracoechea, J. Chem. Phys. 127 (2007) 174103.

[39] K. S. Rane, S. Murali, J. R. Errington, J. Chem. Theory Comput. 9 (2013) 2552-2566.

[40] A. R. V. Koenig, C. Desgranges, J. Delhommelle, Molec. Simul. 40 (2014) 71-79. 
[41] E. A. Hicks, C. Desgranges, J. Delhommelle, Molec. Simul. 40 (2014) 656-663.

[42] A. N. Owen, C. Desgranges, J. Delhommelle, Fluid Phase Equil. 402 (2015) 69-77.

[43] C. Desgranges, J. Delhommelle, J. Chem. Theory Comput. 11 (2015) 5401.

[44] N. B. Vargaftik, Y. K. Vinoradov, V. S. Yargin, Handbook of Physical Properties of Liquids and Gases, Begell House, New York, 1996.

[45] G. C. Boulougouris, I. G. Economou, D. N. Theodorou, J. Phys. Chem. B 102 (1998) 1029-1035.

[46] M. Ceriotti, W. Fang, P. G. Kusalik, R. H. McKenzie, A. Michaelides, M. A. Morales, T. E. Markland, Chem. Rev. (2016). 